\title{
THE AGE, MASS, AND SIZE DISTRIBUTIONS OF STAR CLUSTERS IN M51
}

\author{
Rupali Chandar $^{1}$, Bradley C. Whitmore ${ }^{2}$, Daiana Dinino ${ }^{3}$, Robert C. Kennicutt ${ }^{4}$, L.-H. Chien ${ }^{5}$, \\ Eva SCHINNERER ${ }^{6}$, AND SHARON MeidT ${ }^{6}$ \\ ${ }^{1}$ Department of Physics \& Astronomy, The University of Toledo, Toledo, OH 43606, USA; Rupali.Chandar@utoledo.edu \\ ${ }^{2}$ Space Telescope Science Institute, Baltimore, MD 21218, USA \\ ${ }^{3}$ CICLOPS, Space Science Institute, 4750 Walnut Street, Boulder, CO 80301, USA \\ ${ }^{4}$ Institute of Astronomy, Cambridge University, Cambridge, UK \\ ${ }^{5}$ Department of Physics \& Astronomy, Northern Arizona University, NAU Bo 6010, Flagstaff, AZ 86011, USA \\ ${ }^{6}$ Max-Planck-Institut für Astronomie, Königstuhl 17, D-69117 Heidelberg, Germany \\ Received 2015 September 18; accepted 2016 March 22; published 2016 June 14
}

\begin{abstract}
We present a new catalog of 3816 compact star clusters in the grand design spiral galaxy M51 based on observations taken with the Hubble Space Telescope. The age distribution of the clusters declines starting at very young ages, and can be represented by a power law, $d N / d \tau \propto \tau^{\gamma}$, with $\gamma=-0.65 \pm 0.15$. No significant changes in the shape of the age distribution at different masses is observed. The mass function of the clusters younger than $\tau \approx 400 \mathrm{Myr}$ can also be described by a power law, $d N / d M \propto M^{\beta}$, with $\beta \approx-2.1 \pm 0.2$. We compare these distributions with the predictions from various cluster disruption models, and find that they are consistent with models where clusters disrupt approximately independent of their initial mass, but not with models where lower mass clusters are disrupted earlier than their higher mass counterparts. We find that the half-light radii of clusters more massive than $M \approx 3 \times 10^{4} M_{\odot}$ and with ages between 100 and $400 \mathrm{Myr}$ are larger by a factor of $\approx 3-4$ than their counterparts that are younger than $10^{7}$ years old, suggesting that the clusters physically expand during their early life.
\end{abstract}

Key words: galaxies: individual (M51) - galaxies: starburst - galaxies: star clusters: general - stars: formation

Supporting material: machine-readable table

\section{INTRODUCTION}

The observed age, mass, and size distributions of a population of star clusters provide important clues to their formation, evolution, and dissolution. On galaxy scales, the "initial" cluster mass function (CMF, for clusters younger than $\tau \lesssim 10^{7}$ years) appears to have a fairly similar shape in several galaxies of different type, mass, and interaction stage (e.g., Fall \& Chandar 2012). The CMF/star formation rate (SFR) statistic, which is the initial CMF divided by the total SFR in a galaxy, is very similar in these galaxies, suggesting that the formation rates of stars and clusters are approximately proportional to one another (Chandar et al. 2015). Where they have been studied, the shapes of the cluster age distributions at different masses and the mass functions at different ages appear to be similar, which suggests that the disruption histories of these clusters may also be similar in different galaxies (e.g., Fall \& Chandar 2012; Chandar et al. 2015).

M51 is a nearby, grand-design spiral with a rich population of star clusters that has played a critical role in understanding cluster formation and evolution (e.g., Boutloukos \& Lamers 2003; Bastian et al. 2005; Gieles et al. 2005; Scheepmaker et al. 2007; Hwang \& Lee 2010; Chandar et al. 2011). In this work we present a catalog of compact star clusters selected from the beautiful mosaic images taken with the WFC camera on the Advanced Camera for Surveys (ACS). We use this cluster catalog to determine the age, mass, and size distributions of the clusters, and discuss these results in the context of cluster formation, evolution, and disruption.

The remainder of this paper is organized as follows. Section 2 presents the Hubble Space Telescope (HST) observations of M51 and describes our cluster selection procedure, including estimates of cluster sizes. In Section 3 we derive the age and mass for each star cluster in our sample, including comparisons when different filter combinations are used. Section 4 presents the distributions of cluster masses, ages, and sizes. In Section 5, we discuss the implications of these distributions for the formation, evolution, and dissolution of the clusters in M51. Finally, we summarize our main conclusions in Section 6.

\section{OBSERVATIONS AND STAR CLUSTER CATALOG IN M51}

\subsection{Data}

M51 was observed in a $2 \times 3$ mosaic $^{7}$ with the Wide Field Channel of the Advanced Camera for Surveys (ACS/WFC) in the $F 435 W$ (" $B$ "), $F 555 W$ (" $V$ "), $F 814 W$ (" $I$ ") and the $F 658 N$ ("H $\alpha$ ") filters as part of program GO-10452 (PI: S. Beckwith). The pixel scale of these observations is $0 . .05 \mathrm{pix}^{-1}$, or $2 \mathrm{pc} \mathrm{pix}^{-1}$ at the assumed distance of $8.4 \mathrm{Mpc}$ for M51 (distance modulus $m-M=29.62$; Feldmeier et al. 1997; Vinko et al. 2012). The well publicized color image resulting from this program, available for download from the Hubble Heritage website (http://hubblesite.org/gallery/album/ pr2005012a/), is shown in Figure 1.

We obtained six pointings in M51 with the $F 336 W$ (" $U$ ") filter of the WFPC2 camera as part of program GO-10501 (PI: R. Chandar). Two additional archival $F 336 W$ pointings cover the nuclear region of M51 (GO-5652, PI: R. Kirshner and GO7375, PI: N. Scoville). The raw data were processed through the standard WFPC2 pipeline (CALWP2), and the calibrated images were co-added into a single mosaic image using the

\footnotetext{
7 The observations and resulting mosaic images can be obtained at the following URL: http://archive.stsci.edu/prepds/m51/.
} 


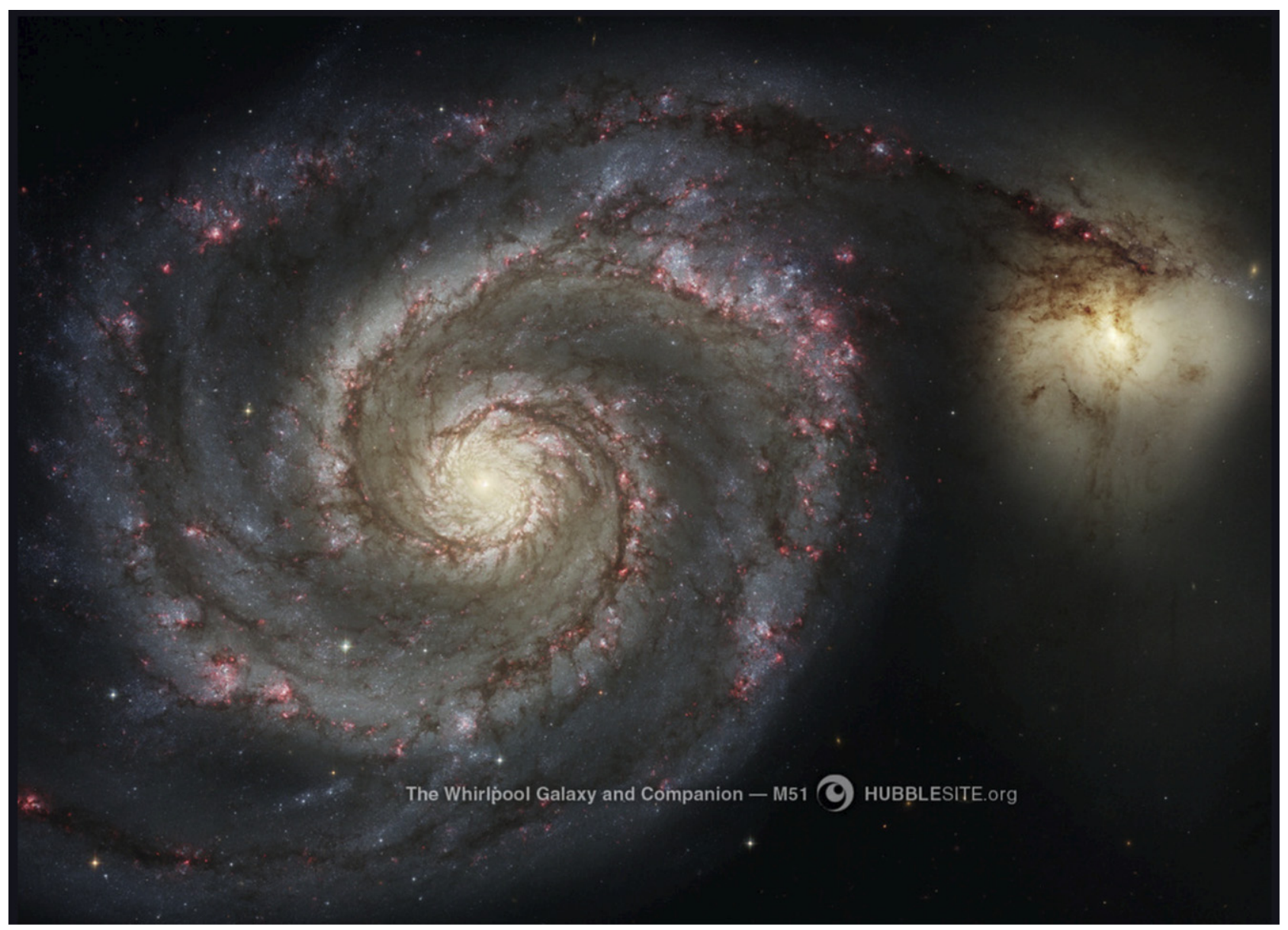

Figure 1. $B V I \mathrm{H} \alpha$ color image of M51 created by M. Mutchler. The observations were taken with the ACS camera on HST.

MULTIDRIZZLE task. Our $U$ band mosaic image is shown in Figure 2, and has a resolution of $0{ }^{\prime \prime} 1 \mathrm{pix}^{-1}$, corresponding to $4 \mathrm{pc} \mathrm{pix}^{-1}$. It covers $\approx 60 \%$ of the luminous portion of the ACS mosaic.

\subsection{Object Detection and Photometry}

We detect sources, both point-like and slightly broader than the point-spread function (PSF), using the IRAF task DAOFIND on a co-added $B, V$, and $I$ band image. Circular aperture photometry was performed on each source in each filter using the IRAF task PHOT, with an aperture radius of 2.5 pixels and background annuli of 10 and 13 pixels. We found that these small apertures gave better photometric results than larger ones. We illustrate this in Figure 3, where the measured $U-B$ versus $V-I$ colors of relatively bright star clusters with $m_{V} \lesssim 21 \mathrm{mag}$ (selected as described below) are compared with the colors predicted from stellar evolution models. The left panel shows measurements using the apertures given above, while the right panel uses a larger aperture of 5 pixels in radius for the $U$ band photometry, similar to that used in Scheepmaker et al. (2009). The larger aperture leads to larger scatter in $U-B$, because flux from nearby sources contaminates the $U$ band measurement for clusters in crowded regions. This scatter is non-symmetric with a red tail, because any contaminating sources that affect the observed colors can only make them redder. The $U$ band photometry was corrected for the effects of charge transfer inefficiency, using the prescription given by Dolphin (2000).

We perform photometry for the narrowband $\mathrm{H} \alpha$ filter from an image where no flux from the stellar continuum was subtracted. This filter contains both stellar continuum (observed for clusters at all ages) and nebular line (observed only for clusters with ages $\tau \lesssim 10 \mathrm{Myr}$ ) emission, which allows us to use this measurement in the spectral energy distribution fits to determine cluster ages and masses of each cluster (Section 3). The instrumental magnitudes were converted to the VEGAMAG photometric system by applying the zeropoints given in Sirianni et al. (2005) for the ACS filters, and those given in Holtzmann et al. (1995) for the WFPC2 filter. We measure the concentration index (CI, defined as the difference in the magnitude measured within a 3 and 0.5 pixel radius) for each cluster, as an estimate of its size. Uncertainties in centering have a very small impact on CI, typically only a few hundredths of a magnitude, since the same center is used to perform photometry in each aperture.

In addition to the CI, we measure the size of each source in our catalog using the Ishape software (Larsen 1999). While the CI index is a very simple method that works well for most clusters, it provides a cruder measure of object size than Ishape. The disadvantage of Ishape is that its size measurements can be less robust in crowded regions and for sources with signal-tonoise $\lesssim 30$, when compared with CI. We use both estimates of cluster size as part of our criteria to select cluster candidates, 


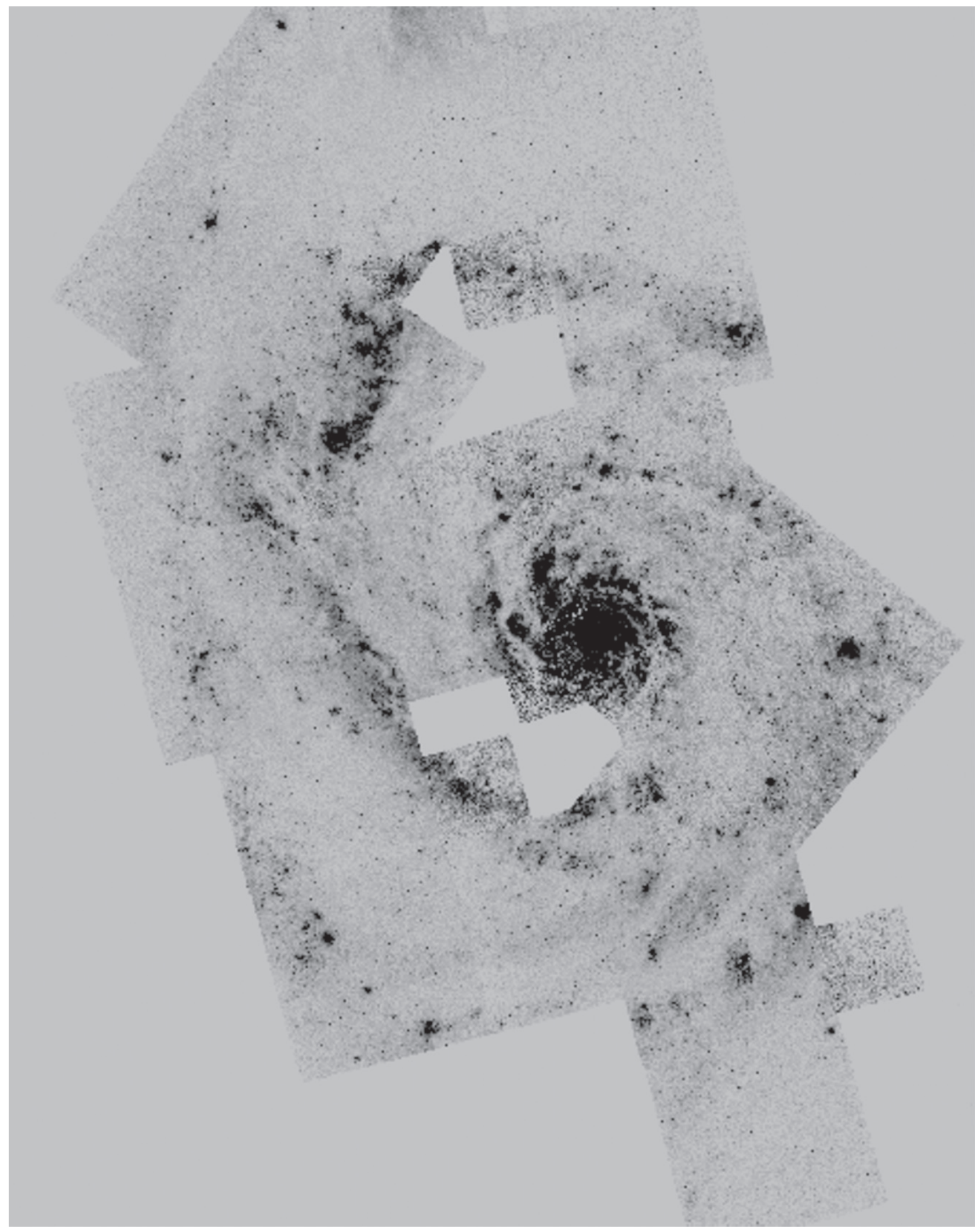

Figure 2. $U$ band mosaic of M51 created using observations taken with the WFPC2 camera on the HST.

and also as a check on the measured sizes, since we are often working in crowded regions.

Ishape convolves analytic profiles of different effective radii, $R_{\text {eff }}$, representing the surface brightness profile of a cluster, with the PSF, and determines the best fit to each source. It returns a measure of the FWHM of a source in pixels, i.e., how much broader a source is than the PSF. In general, Ishape can distinguish sources that are broader than the PSF by $\approx 0.2$ 


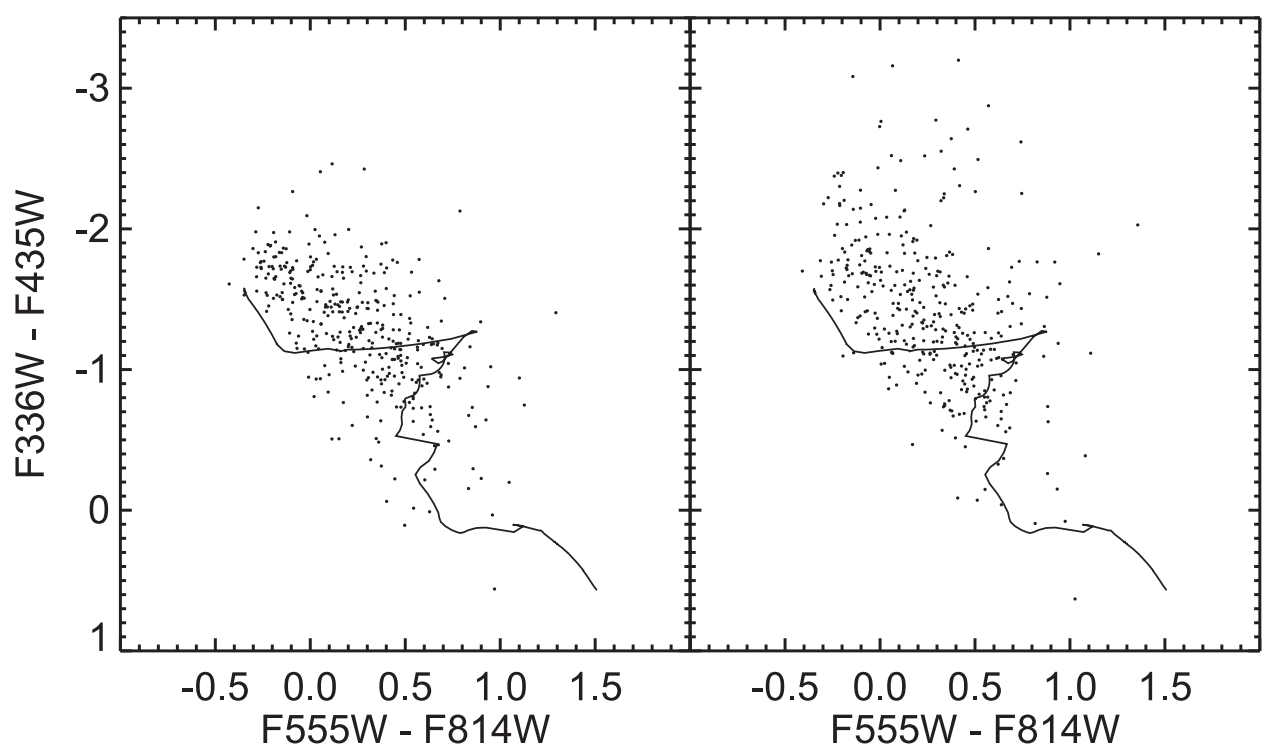

Figure 3. Two-color diagram showing the result of using different size apertures for the WFPC $2 / F 336 W(\approx U)$ band photometry. The left panel uses an aperture of 2.5 pixels and the right panel uses an aperture of 5 pixels for this filter. The larger scatter observed in the right panel is caused by clusters located in crowded regions. A smaller aperture is preferred for these sources. The solid line in each panel shows the predicted colors of clusters with different ages $\left(\tau=10^{6}\right.$ years in the upper left, $\tau=10^{10}$ years in the lower right) from the G. Bruzual \& S. Charlot (2007, private communication) stellar population model with solar metallicity.

pixels or more (e.g., Larsen 1999). We created a PSF for the $V$ band image from $\approx 30$ relatively bright, isolated point sources, and assume a King profile (King 1966) with a ratio of tidal to core radius of 30. Ishape returns the best fit radius of each source, where the fit is performed within a 5 pixel radius.

\subsection{Cluster Selection}

In order to separate star clusters from individual stars and blends of two or more stars, we follow the general approach described in Whitmore et al. (2010) and Chandar et al. (2010b), where we construct "training sets" of stars and clusters and use their measured properties to guide the criteria used to automatically select clusters. Figure 4 shows the separation of isolated stars from young, intermediate-age, and old globular clusters that were selected by hand. The top panel shows the distribution of $M_{V}$ versus CI for our training sets. The stars have the smallest CI values, and separate more-or-less cleanly from our hand-selected clusters brighter than $M_{V} \approx-7$. There also appears to be a trend in the sizes of the clusters, where the CI value of clusters increases with age. This is discussed in Section 4.3. The bottom panel of Figure 4 shows the $U-B$ versus $V-I$ two-color distribution of our training set. The stars (open circles) are red supergiants, and fall away from the cluster tracks but are well matched to stellar tracks (see for example, Figure 7 in Chandar et al. 2010b). Note that many of the stars are too faint to have $U$ band measurements and so are not shown in this figure. The colors of the clusters clearly follow the model predictions.

Based on our training sets, we use the following criteria to select cluster candidates in M51: (i) $m_{V}$ brighter than $23.5 \mathrm{mag}$ (i.e., $\quad M_{V} \lesssim-6$ ); (ii) $1.1 \leqslant C \leqslant 2.0 ; \quad$ (iii) 0.2 pix $\leqslant$ FWHM $\leqslant 5.0$ pix; (iv) eliminate sources that satisfy (i) and (ii) but have another detection within 2 pixels; and (v) select the brightest object within a 5 pixel radius that satisfies (ii) and (iii). We found that critieria (iv) significantly reducted contamination from pairs of close stars, and criteria (v) eliminated detections of of individual stars that are part of stellar clusters. We eliminated remaining, obvious blends from our cluster catalog via visual inspection, as done in recent works (e.g., Bastian et al. 2012; Chandar et al. 2014), resulting in a final catalog of $\sim 3800$ compact stellar clusters. Our full cluster catalog is available online, and the first several entries are shown in Table 1.

Our criteria are designed to select centrally concentrated star clusters, and we do not include less concentrated star forming regions, such as $\mathrm{OB}$ associations. Figure 5 shows two very different stellar clusterings from the Local Group, along with a simulation of how they would look at the distance of M51: the $3 \mathrm{Myr}, \sim 10^{5} M_{\odot}$ cluster R136 is in the LMC in the top left panel, and the diffuse M33 stellar association NGC 406 is in the bottom left panel. The right hand panels show that even at the much larger distance of M51, these two stellar clusterings appear quite different from one another. We exclude diffuse associations from our catalog.

We use two different approaches to determine aperture corrections for the clusters, which convert the fixed aperture magnitudes to total magnitudes. The first approach is to apply a single value of the aperture correction of $0.9 \mathrm{mag}$ in the $V$ band to all clusters, based on empirical measurements of $\sim 30$ relatively isolated, high signal-to-noise ratio $(\mathrm{S} / \mathrm{N})$ star clusters. In this approach, we overestimate the total luminosity of more compact clusters and underestimate the total luminosity of clusters that are more extended. We also apply size-dependent aperture corrections to each cluster, based on their measured CI value. As in Chandar et al. (2010b), we determine a relationship between the aperture correction and CI value for clusters, using measurements of artificial clusters added to the M51 image. While this approach is better in principle, it does not work well for clusters with poorly determined values of $\mathrm{CI}$, either because they have low $\mathrm{S} / \mathrm{N}$ or because they are in very crowded regions.

We assess the completeness of our sample by adding artificial clusters throughout the image, and then subjecting 

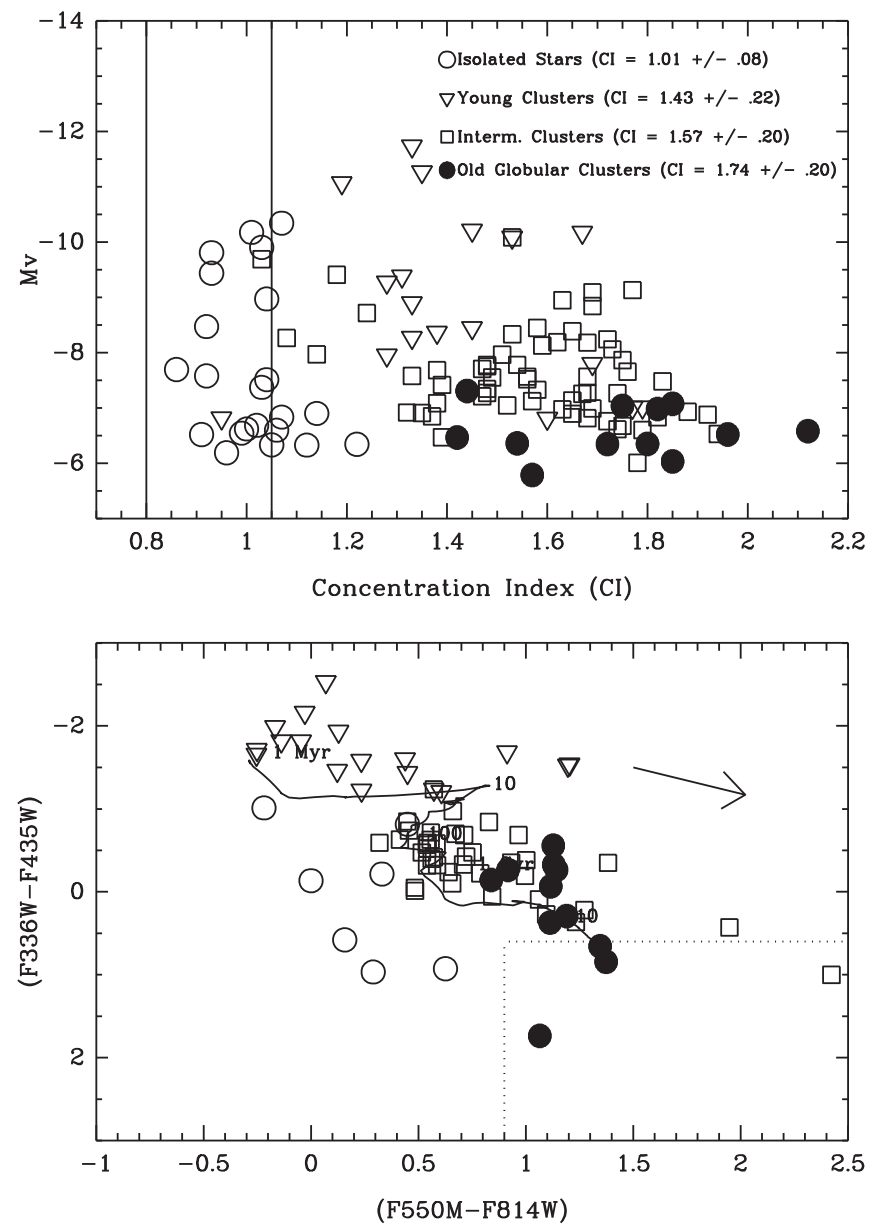

Figure 4. Training set of hand-selected red supergiant stars (open circles) and three types of clusters: (1) young ( $\tau \lesssim 10^{7}$ years) clusters with $\mathrm{H} \alpha$ emission (open triangles), $(2)$ blue, intermediate age $\left(\tau \approx 10^{8}\right.$ years) clusters (open squares), and (3) ancient globular star clusters (filled circles). The upper panel shows the measured concentration index vs. $M_{V}$ for the training objects, and the lower panel shows a two-color diagram for the objects in our training set. The solid track in the lower panel shows the predicted evolution from Bruzual \& Charlot (2007) of star clusters from an age of $1 \mathrm{Myr}$ in the upper left to $10 \mathrm{Gyr}$ in the lower right.

them to our selection criteria. These experiments indicate that our sample is fairly complete (at the $\approx 90 \%$ level) across most of M51. A similar level of completeness occurs closer to $m_{V} \approx 23.0 \mathrm{mag}\left(M_{V} \approx-6.5\right)$ in the most crowded portions of the spiral arms, but these are a small fraction of the total area and do not affect the main conclusions of this work.

We assess the quality of our catalog by comparing with a catalog of clusters selected manually from more recent $H S T$ / WFC3 images of M51, which were taken as part of the LEGUS project (Calzetti et al. 2015). Overall, the cluster catalogs match fairly well at the bright end, but some differences emerge at the faint end. In uncrowded regions, we find that our catalog matches the manual one at the $\approx 70 \%$ level, but that we miss some of the faintest clusters. In crowded regions, the better quality of the WFC 3 observations indicates that while we do not miss many obvious clusters and the overlap is $\approx 60 \%-70 \%$, our current catalog includes contaminants, primarily at the faint end, in the form of blends of two or more stars.

\section{CLUSTER MASS AND AGE ESTIMATES}

\subsection{Determinations Using UBVIH\& Filters}

Age and mass are two basic properties of a star cluster. We estimate the age $\tau$ and extinction $A_{V}$ for each cluster by performing a least $\chi^{2}$ fit comparing observed magnitudes with the predictions from G. Bruzual \& S. Charlot (2007, private communication, see also Bruzual \& Charlot 2003) single stellar population models assuming solar metallicity $Z=0.02$, a Salpeter (1955) IMF, and a Galactic-type extinction law (Fitzpatrick 1999). The best-fit values of $\tau$ and $A_{V}$ are those that minimize the statistic

$$
\chi^{2}\left(\tau, A_{V}\right)=\sum_{\lambda} W_{\lambda}\left(m_{\lambda}^{\text {obs }}-m_{\lambda}^{\text {mod }}\right)^{2},
$$

where $m_{\lambda}^{\text {obs }}$ and $m_{\lambda}^{\text {mod }}$ are the observed and model magnitudes respectively, and the sum runs over all five bands, $\lambda=U, B, V, I$, and $F 658 \mathrm{~N}$. The weight factors in the formula for $\chi^{2}$ are taken to be $W_{\lambda}=\left[\sigma_{\lambda}^{2}+(0.05)^{2}\right]^{-1}$, where $\sigma_{\lambda}$ is the formal photometric uncertainty determined by PHOT for each band. The mass of each cluster is estimated from the observed $V$ band luminosity, corrected for extinction, and the (presentday) age-dependent mass-to-light ratios $\left(M / L_{V}\right)$ predicted by the models, assuming a distance modulus $\Delta(m-M)$ of 29.62 or $8.4 \mathrm{Mpc}$ for M51 (Feldmeier et al. 1997; Vinko et al. 2012). ${ }^{8}$

\subsection{Comparison of Ages Determined from Different Filter Combinations}

The majority of clusters in our sample have measurements in all five $U B V I H \alpha$ filters. However, we only require measurements in three filters, including the $V$ band, to estimate the age (and mass) of a star cluster. This means that we also estimate ages (albeit with larger uncertainties) for the few clusters in our sample that do not have photometry in the $U$ and/or in the narrow band filter, either due to lack of coverage or because they are too faint in one of these filters.

A comparison of our photometric age determinations and previous spectroscopic ones (presented below) indicates that the UBVI,H $\alpha$ filter combination returns robust age determinations. Here, we compare the differences in the age estimates when two other filter combinations are used: $U B V I$ (i.e., no $\mathrm{H} \alpha$ ), which is the most common filter combination used in the literature for estimating the ages of star clusters, and $B V I, \mathrm{H} \alpha$ (i.e., no $U$ band), which was used here for clusters when no $U$ band imaging is available. We assume the same metallicity models, extinction law, etc. as used for the age estimates based on the full $U B V I, \mathrm{H} \alpha$ filter set.

The left panel of Figure 6 compares ages determined from the $U B V I \mathrm{H} \alpha$ and $U B V I$ filter combinations, i.e., with and without the narrowband $\mathrm{H} \alpha$ filter. We find that including the narrowband filter significantly improves the age determinations for $\approx 35 \%$ of the clusters, those affected by the age-extinction degeneracy where it is not clear if the clusters are quite young but extincted, or older and gas-free. Approximately $65 \%$ of the clusters in this figure have age estimates that are within 0.3 in $\log \tau$ of one another (this is the typical uncertainty in age estimates made by comparing integrated cluster colors with stellar evolution models, e.g., de Grijs \& Anders 2006;

\footnotetext{
8 Adopting a shorter (longer) distance to M51 will reduce (increase) the derived cluster masses, but does not affect any of the main conclusions of this paper.
} 
Table 1

Catalog of Star Clusters in M51

\begin{tabular}{|c|c|c|c|c|c|c|}
\hline $\begin{array}{l}\text { ID } \\
\#\end{array}$ & $\begin{array}{c}x \\
H S T\end{array}$ & $\begin{array}{c}y \\
H S T\end{array}$ & $\begin{array}{l}\text { R.A. } \\
\text { (deg) }\end{array}$ & $\begin{array}{l}\text { decl. } \\
(\operatorname{deg})\end{array}$ & $\begin{array}{c}\log \text { Age } \\
\log \left(\tau \mathrm{yr}^{-1}\right)\end{array}$ & $\begin{array}{c}\log \text { Mass } \\
\log \left(M / M_{\odot}\right)\end{array}$ \\
\hline 1 & 7578.64 & 79.41 & 202.4004112 & 47.1302503 & 8.06 & 4.29 \\
\hline 2 & 7332.76 & 88.68 & 202.4054309 & 47.130382 & 6.78 & 3.11 \\
\hline 3 & 4768.15 & 237.07 & 202.457789 & 47.132461 & 6 & 3.86 \\
\hline 4 & 4608.48 & 239.43 & 202.4610489 & 47.1324941 & 6.78 & 4.16 \\
\hline 5 & 5476.04 & 263.77 & 202.4433361 & 47.1328292 & 9.23 & 5.18 \\
\hline
\end{tabular}

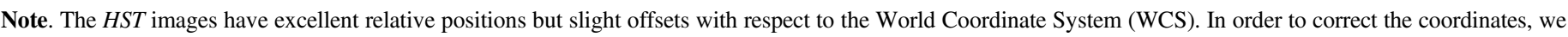
have added -0.0000560 to the R.A. and -0.1930 to the decl.

(This table is available in its entirety in machine-readable form.)
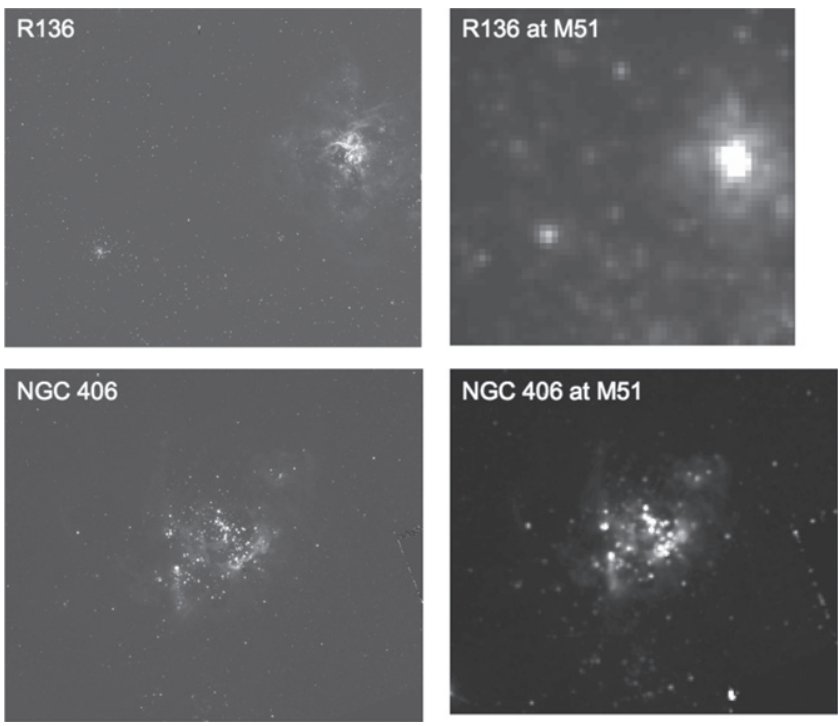

Figure 5. Ground-based, $V$-band image of compact cluster R136 (top-left) and its surrounding association in the Large Magellanic Cloud, and an HST WFPC2 image of diffuse stellar association NGC 406 in M33 (bottom-left). The simulations in the right panels of how R136 and NGC 406 would appear in M51 as observed with the ACS/WFC, indicates that we can tell the difference between compact clusters and diffuse stellar associations.

Chandar et al. 2010a and later in this section). The remaining $35 \%$ are found in one of two "chimneys" (to use the nomenclature of Scheepmaker et al. 2009) where clusters have inconsistent ages between the two filter sets, one where inclusion of $\mathrm{H} \alpha$ measurements results in older ages ( $\tau>10^{7}$ years; labeled "A") and the other where including $\mathrm{H} \alpha$ gives ages $\tau<10^{7}$ years (labeled "B").

We examined clusters in these two categories. Clusters in "B" are found mostly in very crowded regions along the spiral arms, and many are $\mathrm{H}$ II regions, indicating that these are young objects with $\tau \lesssim 10^{7}$ years. The location of these clusters in the two-color diagram is somewhat ambiguous as to whether they are young and moderately reddened objects, or slightly older clusters with little extinction. The presence of nebular emission indicates that these are moderately reddened young ( $\tau<10^{7}$ years) clusters, consistent with their location along the spiral arms. Clusters in chimney "A" also fall in a degenerate portion of the two-color diagram. We find that these clusters are preferentially located somewhat away from spiral arms, and clearly do not contain $\mathrm{H} \alpha$ emission. Therefore, we believe that these objects are somewhat older than $\approx 10^{7}$ years.
The lack of nebular emission drives the $U B V I, \mathrm{H} \alpha$ fit toward older ages, whereas the $U B V I$ filter combination has trouble breaking the degeneracy between age and extinction for these objects.

The $B V I, \mathrm{H} \alpha$ filter combination plays an important role in our analysis of star clusters in M51, because this combination allows us to estimate the ages of clusters in regions where no $U$ band imaging exists. The right panel of Figure 6 compares ages estimated with and without the $U$ band. We find that $\approx 75 \%$ of the clusters have age estimates within 0.3 in $\log \tau$, but there is a systematic trend that ages estimated without $U$ band are slightly older. This is because the procedure slightly favors an older age with lower extinction to a younger age with somewhat higher extinction. Overall, the $B V I, \mathrm{H} \alpha$ filter combination gives robust age determinations for most star clusters which do not have $U$ band photometry available. For all of the reasons described above, we strongly advocate that whenever possible, authors include a measure of the $\mathrm{H} \alpha$ line or some other appropriate narrowband filter when estimating the age of star clusters.

We estimate the typical uncertainties in the age and mass estimates, and find them to be similar to those suggested by previous works (e.g., de Grijs \& Anders 2006, Chandar et al. 2010a). We did this by estimating ages and masses from our $\chi^{2}$ analysis for different filter combinations (as described above), different assumptions regarding extinction (using the Calzetti et al. 1994 obscuration curve), and different metallicities (also used lower metallicity model $Z=0.008$ ). Based on all of our experiments, we find typical $1 \sigma$ uncertainties of $\approx 0.3$ in $\log \tau$. We believe that this is a reasonable estimate of the uncertainties in the ages, based on the benchmarking of age determinations done below. This level of uncertainty is common for age estimates obtained by comparing integrated colors with model predictions (similar levels of uncertainty were estimated for clusters in the Antennae by Fall et al. 2005 and in the Magellanic Clouds by de Grijs \& Anders 2006 and Chandar et al. 2010a). We note that age and metallicity become degenerate for clusters older than $\approx 10^{9}$ years, particularly because the young clusters in M51 have near-solar metallicity, while the ancient globular clusters appear to be mostly metalpoor (Chandar et al. 2004).

The random uncertainties in the age translate to $1 \sigma$ uncertainties of $\approx 0.3$ in $\log M$ or a factor of two in $M$. There are also systematic uncertainties in the mass determinations, from the assumed stellar IMF and from the assumed distance to M51. If we had assumed a Chabrier (2003) rather than a Salpeter (1955) stellar IMF for each cluster, the $M / L_{V}$ and hence the mass of each cluster would be reduced by an (ageindependent) $40 \% \quad$ (e.g., see discussion in Chandar 


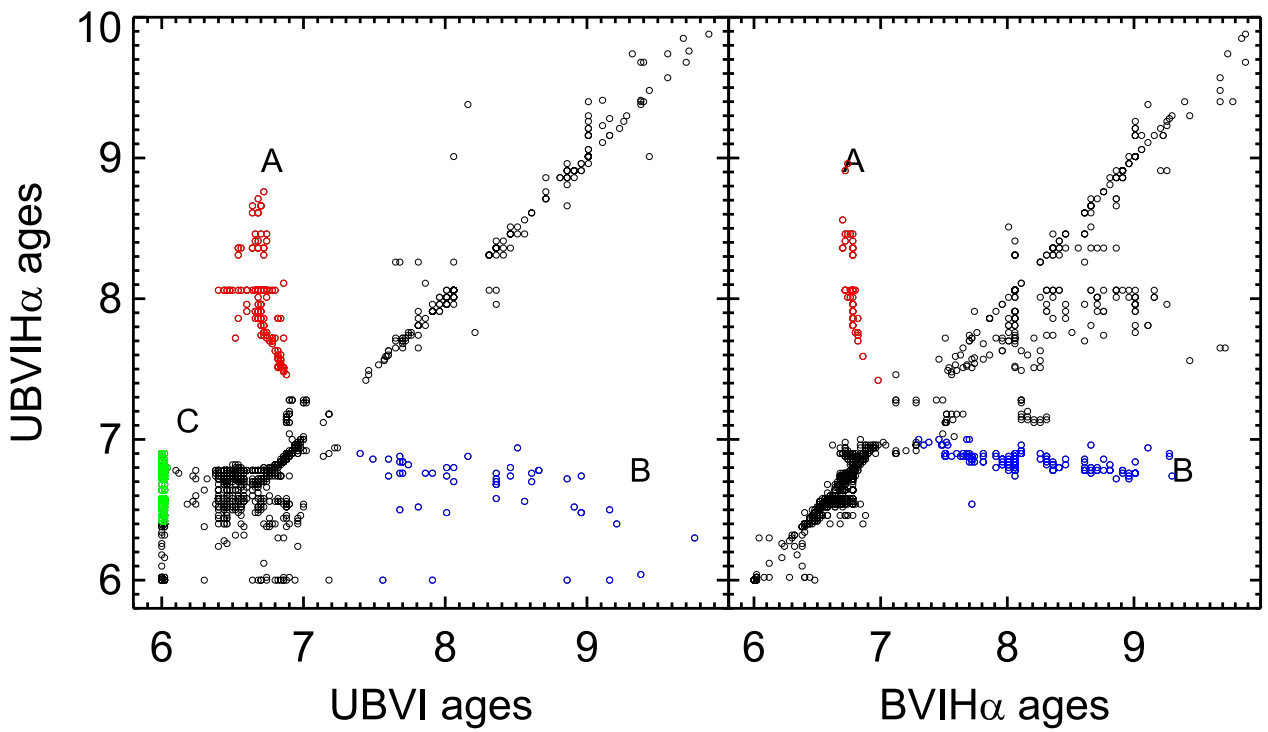

Figure 6. Comparison of ages (in $\log \left(\tau \mathrm{yr}^{-1}\right)$ derived for clusters in M51 using different filter combinations. The left panel shows $U B V I \mathrm{H} \alpha$ vs. $U B V I$, and the right panel show $U B V I \mathrm{H} \alpha$ vs. $B V I \mathrm{H} \alpha$. Clusters located in the regions labeled "A," "B," and "C" have mismatches in ages from the different filter combinations, and are discussed in the text.

et al. 2010a). Adopting a shorter (longer) distance to M51 would also systematically reduce (increase) the derived masses of the clusters. However, these systematic effects do not affect the shape of the CMF. In fact, our experiments show that the CMF is surprisingly robust to different filter combinations and age-dating techniques, and to different stellar evolution models, extinction laws, and metallicity.

\subsection{Comparison of Photometric and Spectroscopic Age Determinations}

In Table 2 we compare our age estimates with those determined by Bastian et al. (2008) for six clusters in M51 using integrated spectroscopy from Gemini-North. We also present ages determined from the $U B V I$ and $B V I \mathrm{H} \alpha$ filter combinations for these clusters as illustrative examples of the systematic differences that can result when the $U$ or $\mathrm{H} \alpha$ are missing. All of the clusters in this sample are fairly young with $\tau \lesssim 2 \times 10^{7}$ years, and overall our age estimates are in good agreement with those determined spectroscopically. Only cluster $\mathrm{G} 2 \mathrm{~b}$ has a photometric age determination from the $U B V I \mathrm{H} \alpha$ combination which is formally outside of the uncertainties, when compared with its spectroscopic age. G2b has a too-large age determination from the UBVI filter combination because without $\mathrm{H} \alpha$ the software assumes that the redder colors are due to an older age rather than a higher extinction. Similarly, the $B V I \mathrm{H} \alpha$ combination returs a somewhat older age for cluster 3c1-a. More quantitatively, the mean offset between our preferred age determinations and those based on spectroscopy is only $0.6 \mathrm{Myr}$, with a standard deviation of 3 Myr. Our photometric age determinations without the $U$ band filter, using only the $B V I \mathrm{H} \alpha$ filter measurements, are fairly similar to those when this filter is included, with an age difference of $-2 \pm 6$ Myr. Ages determined from the $U B V I$ filter combination give the poorest match to the spectroscopic ages, with three out of six clusters (a1, G2a, G2b) having photometric age estimates that do not match the spectroscopic ages within the uncertainties, and with a mean difference for this sample of $-6 \pm 20 \mathrm{Myr}$. This is primarily due to the fact that it is more difficult to break the
Table 2

Comparison between Photometric and Spectroscopic Age Estimates for Clusters in M51

\begin{tabular}{|c|c|c|c|c|}
\hline $\begin{array}{l}\text { Cluster } \\
\text { ID }\end{array}$ & $\begin{array}{c}\text { Spectroscopic }^{\mathrm{a}} \\
\text { Age (Myr) }\end{array}$ & $\begin{array}{c}U B V I \mathrm{Ha}^{\mathrm{b}} \\
\text { Age (Myr) }\end{array}$ & $\begin{array}{c}U B V I^{\mathrm{b}} \\
\text { Age (Myr) }\end{array}$ & $\begin{array}{c}B V I \mathrm{Ha}^{\mathrm{b}} \\
\text { Age }(\mathrm{Myr})\end{array}$ \\
\hline a1 & $5(4,8.9)$ & 6 & 1 & 6 \\
\hline $3 \mathrm{c} 1-\mathrm{a}$ & $16.5(12.6,25.1)$ & 13 & 8 & 30 \\
\hline $3 \mathrm{c} 1-\mathrm{b}$ & $5(4,6)$ & 9 & 10 & 9 \\
\hline $2 \mathrm{c} 1-\mathrm{c}$ & $3(2,5)$ & 1 & 5 & 1 \\
\hline G2a & $6(4,14)$ & 7 & 1 & 6 \\
\hline $\mathrm{G} 2 \mathrm{~b}$ & $5(3,7)$ & 1 & 52 & 1 \\
\hline$\overline{\Delta(\tau)}^{\mathrm{c}}$ & $\ldots$ & $0.6 \pm 3$ & $-6 \pm 20$ & $-2 \pm 6$ \\
\hline
\end{tabular}

Notes.

${ }^{\mathrm{a}}$ Best fit age from analysis of spectra. Upper and lower age estimates are given in parentheses.

${ }^{\mathrm{b}}$ Filter combination used to determine ages. Typical uncertainties are $\log \tau \approx 0.3$ or a factor of two in the age.

${ }^{c}$ The mean and standard deviation of the photometric age estimates minus spectroscopic age estimates from Bastian et al. (2008).

age-extinction degeneracy without the $\mathrm{H} \alpha$ filter at these young ages.

\section{CLUSTER MASS, AGE, AND SIZE DISTRIBUTIONS}

Figure 7 shows the mass-age $(M-\tau)$ diagram of star clusters in M51. We find cluster ages that span the age of the galaxy, although there are few clusters with estimated ages that are less than $\approx 2 \mathrm{Myr}$, since very young clusters remain embedded in their natal molecular cloud until feedback from the massive stars disperses this material. The solid edge along the bottom of the data points shows the luminosity limit of $V=23.5$ that we imposed during the cluster selection procedure. The $M-\tau$ diagram has a number of small-scale features, where clusters pile up at specific ages or avoid others. The lack of clusters between $7.0 \lesssim \log \left(\tau \mathrm{yr}^{-1}\right) \lesssim 7.5$ years, for example, occurs because the predicted colors loop back on themselves, covering a small region in color-color space over a relatively long time, and resulting in a gap. The broad distributions of cluster masses 


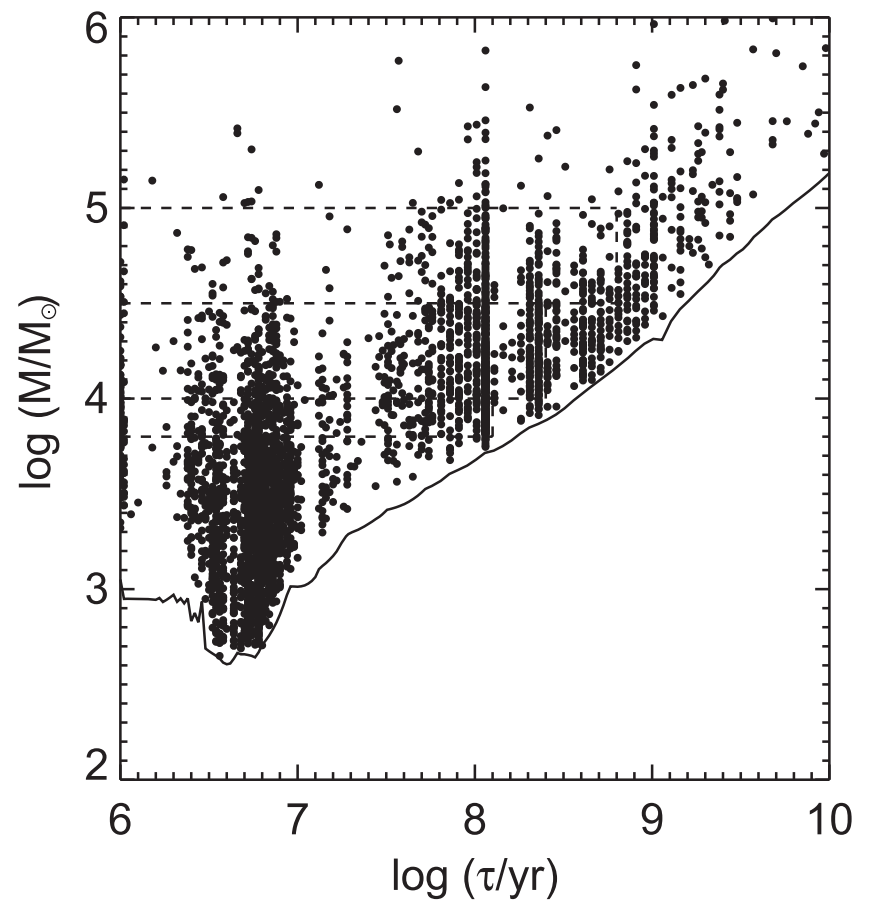

Figure 7. $\log M$ vs. $\log \tau$ derived for clusters in our M51 catalog. The solid line shows the approximate limiting magnitude of our catalog of $M_{V}=-6$. The dashed lines show that the mass-age ranges used for the age distributions stay above this magnitude limit (as do those for the mass functions, not shown).

and ages, however, are not strongly affected by the small-scale features.

Some basic trends in the cluster mass and age distributions are apparent from the $M-\tau$ diagram. When we look along the horizontal axis within a given range of $\log M$, we see that the number of clusters increases slightly in equal bins of $\log \tau$. This suggests that the cluster age distribution can be described, at least approximately, by a power law, $d N / d \tau \propto \tau^{\gamma}$, with $\gamma$ somewhat shallower than -1 , but clearly steeper than 0 ; $\gamma \approx 0$ would result in a strongly increasing number of clusters at older ages (see Figure 3 in Whitmore et al. 2007). When we look down the vertical axis, we see that the number of clusters increases steadily with decreasing mass. This suggests that the CMF can be described, at least approximately, by a power law, $d N / d M \propto M^{\beta}$, with $\beta$ much steeper than -1 , which would give a constant distribution. A quantitative treatment is given in the next two subsections.

\subsection{Age Distributions}

Figure 8 shows the age distribution of the star clusters in M51 in three different intervals of mass: $\log \left(M / M_{\odot}\right)=$ 4.5-5.0, $\log \left(M / M_{\odot}\right)=4.0-4.5$, and $\log \left(M / M_{\odot}\right)=3.8-4.0$. We have used broad bins in $\log \tau$ in order to bridge the smallscale features in the $M-\tau$ diagram, and restricted the plotted range to stay above the luminosity limit of the cluster sample.

The distributions in all three mass ranges decline starting at very young ages, and have best fits between $\gamma=-0.6$ and $\gamma=-0.7$. If we use different mass-age ranges and different binning, we find that all of the values for $\gamma$ are between $\approx-0.5$ and $\approx-0.8$. Based on these experiments, we conclude that the exponent for the age distribution of clusters in M51 is approximately $\gamma=-0.65 \pm 0.15$.

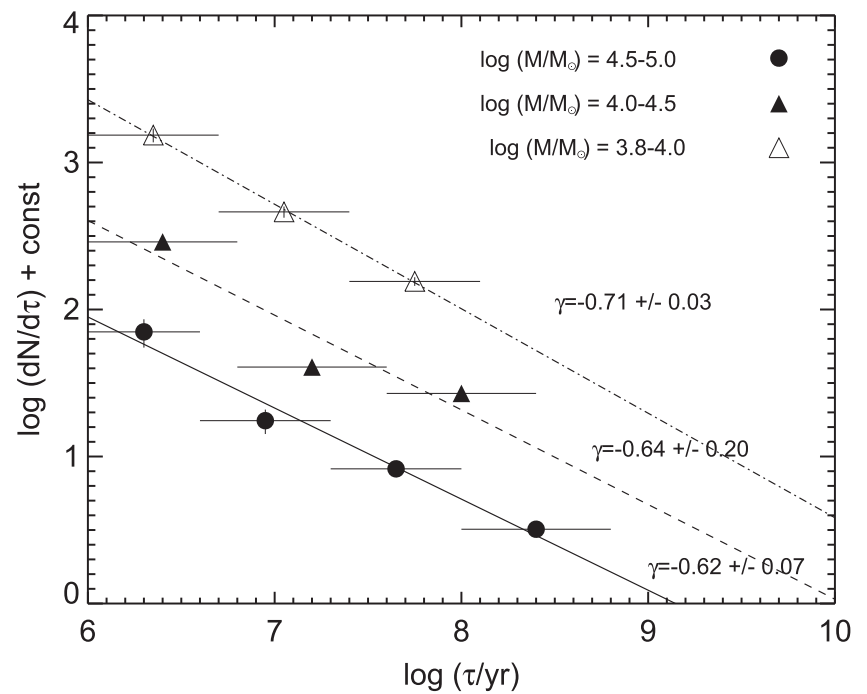

Figure 8. Age distribution of star clusters in M51 in different mass intervals. The normalizations of the age distributions in the vertical direction are arbitrary. The lines show power laws, $d N / d \tau \propto \tau^{\gamma}$, with the best-fit exponents given in the figure.

\subsection{Mass Function}

Figure 9 shows the mass function of star clusters in M51 in three different intervals of age: $\log \tau=6-7, \log \tau=7-8$, and $\log \tau=8-8.6$, based on our dating analysis. Two different binnings are shown, variable size bins with equal numbers of clusters in each bin (filled circles, see Maiz Apellaniz \& Ubeda 2005) and approximately equal size bins with variable numbers of clusters in each bin (open circles). Each distribution is restricted to stay above the cluster selection limit.

The mass functions can be approximated by a power law, $d N / d M \propto M^{\beta}$, and we find best fit values of $\beta$ in the three age ranges of $-2.06 \pm 0.05,-1.97 \pm 0.09$, and $-2.25 \pm 0.06$ in the three different age intervals. The given errors are just the formal uncertainties on the fit. The mass functions do not show an obvious deviation from a power law, such as a bend at either the high or low mass end. The results are not sensitive to the method used to make aperture corrections. If we had assumed a different stellar IMF, the distribution would move to higher or lower masses, but without changing shape. We conclude that the exponent for the mass function of clusters in M51 is approximately $\beta=-2.1 \pm 0.2$.

\subsection{Size Distribution}

In the top panel of Figure 4, our hand-selected training sets of different age clusters suggests that young clusters tend to have smaller values of CI than older clusters. We quantify this trend below.

Figure 10 shows the $V$-band size measurements of massive clusters in M51. We have selected four subsamples, restricting to high cluster masses, where the measurements are likely to be more robust, and to young $\tau<10^{7}$ years and intermediate age ( $\log \tau=8.0-8.6$ years) clusters, the two age ranges which tend to have the most robust age determinations. The left hand panel shows that for almost all clusters, two different size determinations, the FWHM measured from ISHAPE and the CI, result in a relatively tight, although nonlinear correlation. This is shown for two different ranges of mass: $\log M>4.8$ (upper panel) and $\log M=4.5-4.8$ (lower panel). In both 


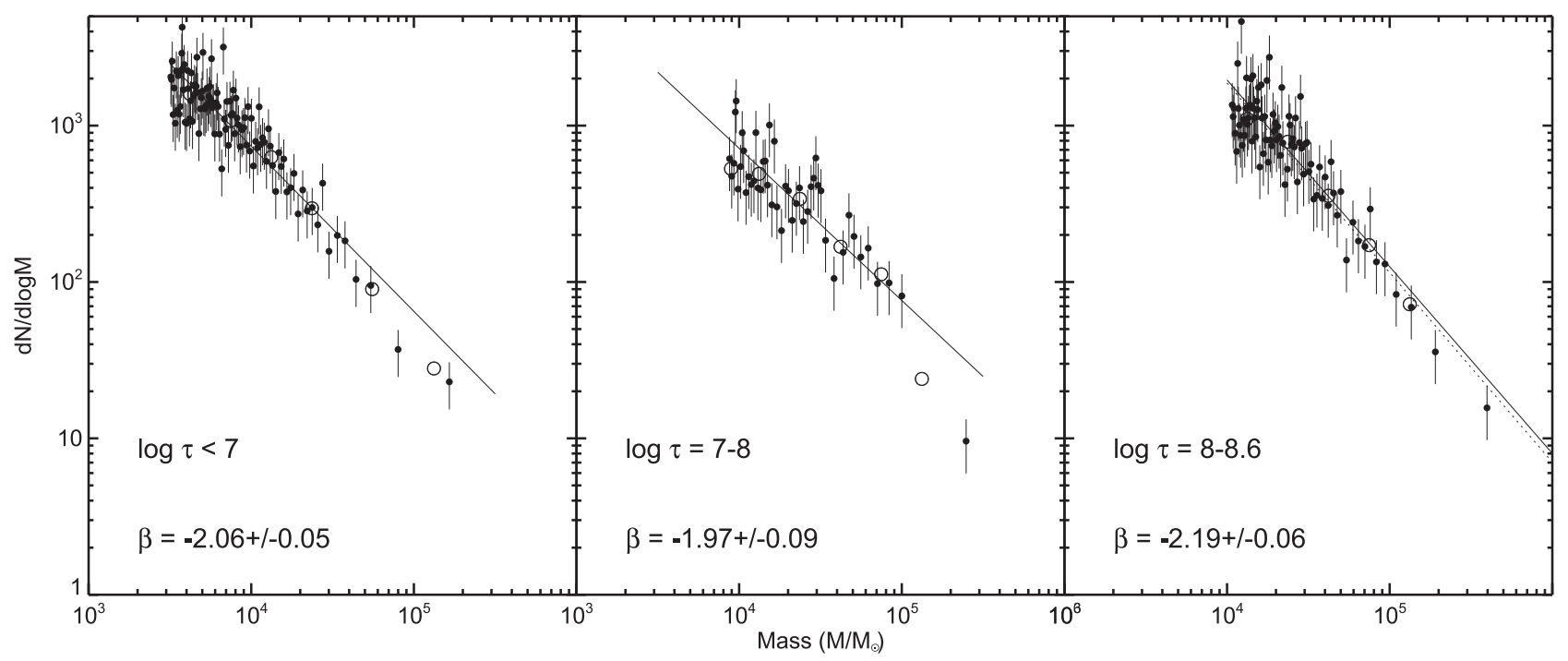

Figure 9. Mass functions of star clusters in M51 in the indicated intervals of age. The data are restricted to stay above the approximate completion limit shown as the solid line in Figure 7. The bins have variable widths but the same number of clusters in each bin (filled circles), or roughly equal widths but variable numbers of clusters (open circles). The lines show power laws, $d N / d M \propto M^{\beta}$, with the best-fit exponents given in the figure.
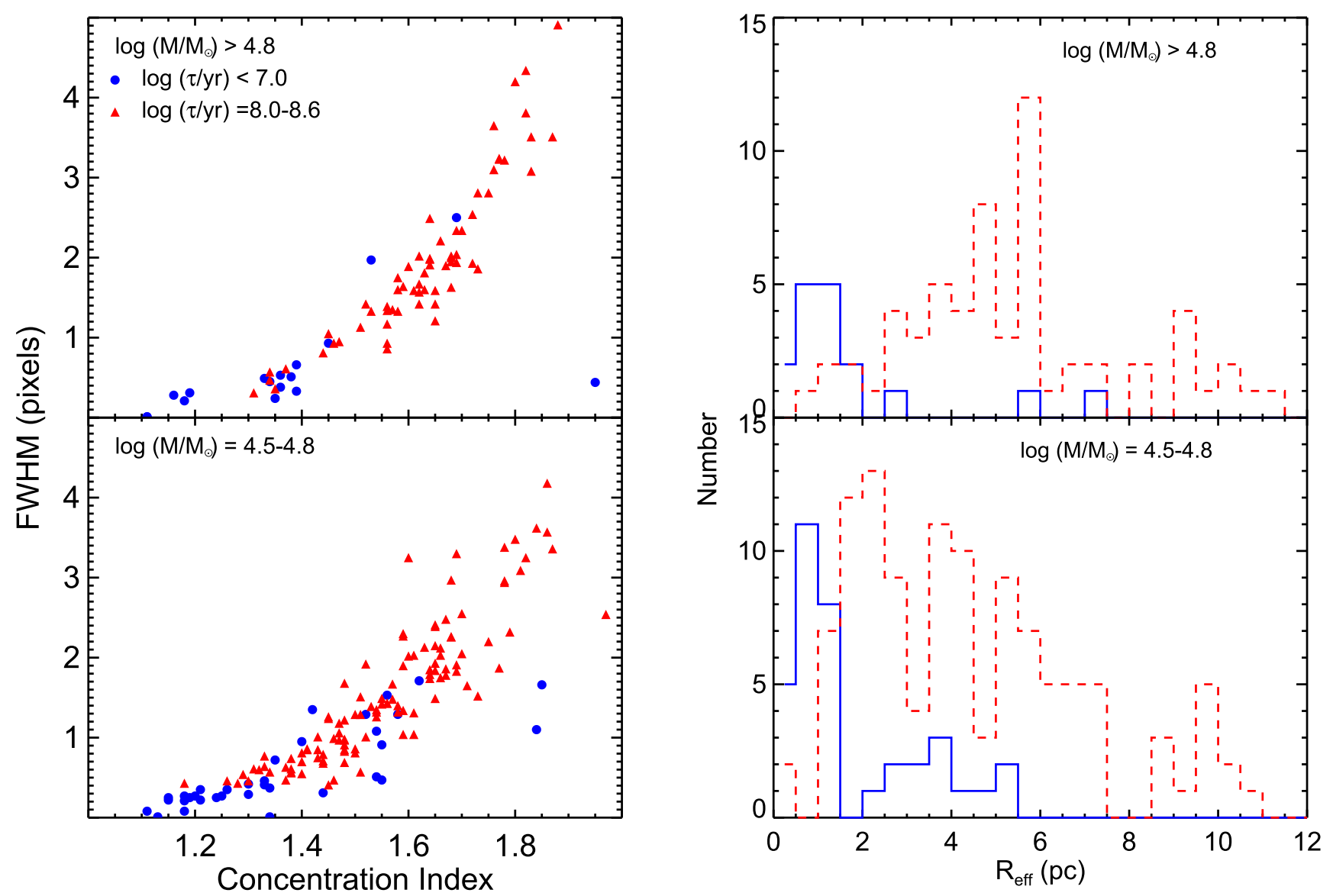

Figure 10. The left panels show that there is a good, albeit nonlinear, correlation between two different size measurements: the concentration index and the FWHM (in pixels) measured by the Ishape software. The top panel shows the measurements for clusters with estimated masses greater than $\log \left(M / M_{\odot}\right)>4.8$, and the bottom panel for clusters with masses $\log \left(M / M_{\odot}\right)=4.5-4.8$. Different symbols are used for clusters younger than $\log \left(\tau \mathrm{yr}^{-1}\right)=7.0$ (blue circles) and those with ages $\log \left(\tau \mathrm{yr}^{-1}\right)=8.0-8.6$ (red triangles). The right panels show the distribution of $R_{\mathrm{eff}}$ from Ishape for the clusters in the higher and lower mass ranges, as well as in the younger (blue) and older (red) age ranges.

cases, CI tracks the FWHM measurements remarkably well, with scatter primarily only for very young clusters in the most crowded regions.
The second (right) set of panels shows the distribution of cluster sizes from Ishape for a given range of age and mass. Clearly, very young clusters are significantly more compact 
than older clusters with similar masses, which is expected based on the properties of our training sets shown in the top panel of Figure 4 . We find median effective radii $R_{\text {eff }}$ of 0.44 pix $(1.3 \mathrm{pc})$ and 0.35 pix $(1.0 \mathrm{pc})$ for clusters with ages $<10$ Myr and masses $\log M>4.8$ and $\log M=4.5-4.8$, respectively. For $100-400$ Myr clusters, we find median sizes of $R_{\text {eff }}=1.86$ pix $(5.5 \mathrm{pc})$ and 1.39 pix $(4.1 \mathrm{pc})$ in the same ranges of mass. Hence, we find that the sizes of star clusters (of similar mass) increase by a factor of $\approx 4$ over the first few hundred million years. We also note that the typical size of more massive clusters at older ages is slightly larger than lower mass clusters, where the best fit gives $M \propto R_{\mathrm{eff}}^{0.26 \pm 0.03}$ over the studied mass range; no obvious trend with cluster mass is observed for clusters with ages younger than $10 \mathrm{Myr}$.

This age-size relationship for clusters in M51 is similar to that found in other works. Previously, Scheepmaker et al. (2007) noted an increase in size for redder clusters in M51, although they were unable to fully quantify the evolution in cluster size because they did not have age estimates for their full cluster sample. Bastian et al. (2008) compiled information on clusters in M51, the Magellanic Clouds and M82, and found a similar trend in the age-size relationship as found here. Using a much larger number of clusters in M83, Bastian et al. (2012) found an increase of approximately a factor of three in cluster $R_{\text {eff }}$ over a few 100 Myr (see their Figure 14), and little trend in cluster size with mass. More recently, Ryon et al. (2015) found a weaker increase in the sizes of M83 clusters over the more limited age range from $\sim 30 \mathrm{Myr}$ to $\sim 300 \mathrm{Myr}$.

\section{DISCUSSION}

The mass, age, and size distributions of star clusters provide some of the primary observational windows into the formation and disruption of the clusters.

The "initial" ( $\tau \lesssim 10 \mathrm{Myr}$ ) mass function of star clusters within a galaxy, which is as fundamental as the initial stellar mass function within a cluster, appears to have a similar shape in many nearby galaxies. In this work, we find that the data for M51 are well fit by a power law with index $\beta \approx-2.1$. This is similar to the mass functions for $\tau \lesssim 10$ Myr clusters in several other star-forming galaxies, including spirals, starbursts, and mergers, where values of $\beta$ have been found to be $\beta=-2.0 \pm 0.3$ (Fall \& Chandar 2012; Chandar et al. 2015).

The shape of the $\mathrm{CMF}$ at intermediate ages, $\tau=100-400 \mathrm{Myr}$ is similar to that of the initial CMF. The low mass end of the $\mathrm{CMF}$ at these older ages provides direct constraints on the disruption of the clusters over this timescale, and in particular whether or not the disruption depends on the initial mass of the clusters. If lower mass clusters disrupt earlier than higher mass clusters then the mass function should flatten toward lower masses, and disruption models (e.g., Lamers et al. 2005; Fall et al. 2009) can be used to determine the characteristic disruption time of a $10^{4} M_{\odot}$ cluster. If no such flattening is observed, then a lower limit to this disruption time can be established. The models assume that the mass $M$ of a cluster evolves gradually over time according to:

$$
\begin{gathered}
d M / d \tau=-M / \tau_{d}(M), \\
\tau_{d}(M)=\tau_{*}\left(M / M_{*}\right)^{k},
\end{gathered}
$$

where the exponent $k$ and the characteristic disruption timescale $\tau_{*}$ are adjustable parameters, while $M_{*}=10^{4} M_{\odot}$ is a fiducial mass scale. Equations (B6)-(B8) in Fall et al. (2009) give the

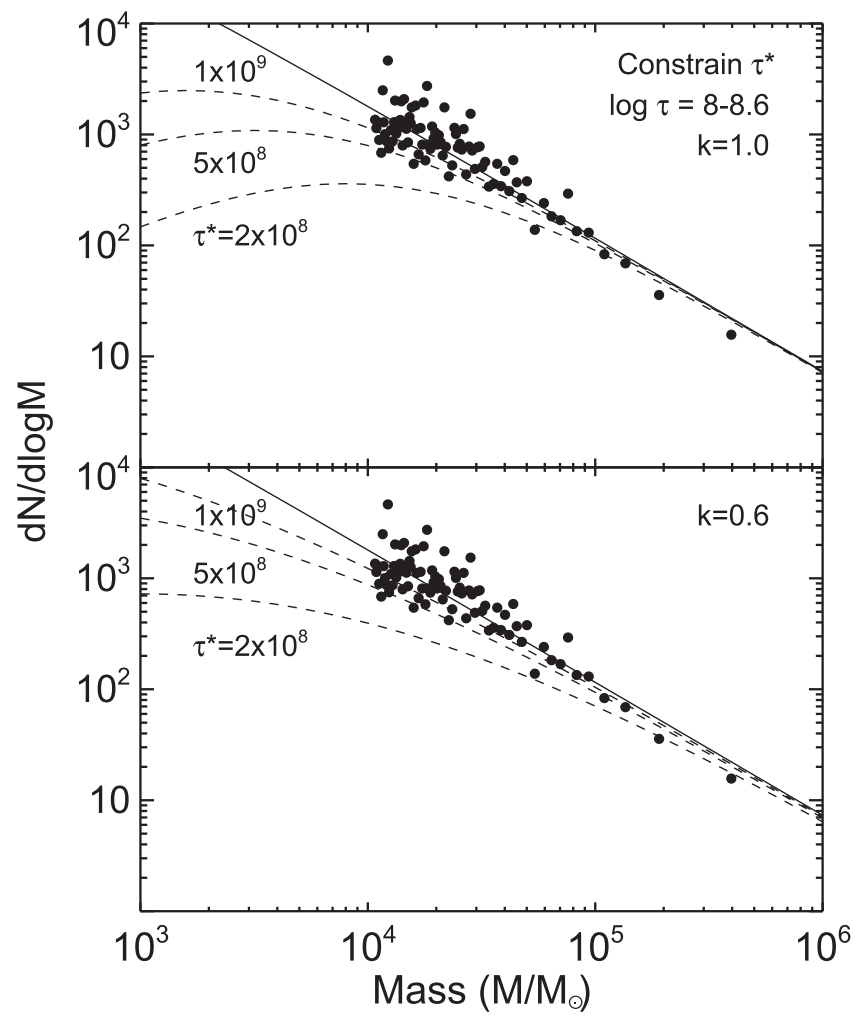

Figure 11. Mass function for $1-4 \times 10^{8}$ years clusters in M51 are compared with gradual disruption models with $k=1.0$ (upper panel) and $k=0.6$ (lower panel), with the indicated disruption timescales $\tau_{*}$. The curves use the Equations (B6)-(B8) from Fall et al. (2009). $\tau_{*}$ values of $5 \times 10^{8}$ years and younger are clearly ruled out in both cases, and for $k=1.0$ a lower limit of $\tau_{*} \gtrsim 10^{9}$ years is indicated by the comparison between the observations and model predictions.

analytic expressions derived for the CMF that can be compared directly with observations.

In Figure 11, we compare the shape of the observed mass function of intermediate age clusters in M51 with predictions for $k=1.0$ (top panel) and $k=0.6$ (bottom panel) with values for $\tau_{*}$ of $2 \times 10^{8}$ years, $5 \times 10^{8}$ years, and $1 \times 10^{9}$ years. The lowest curve in the bottom panel shows that a disruption time of $\tau_{*}=2 \times 10^{8}$ years for $k=0.6$, the values suggested by Gieles et al. (2005) for M51, predict significantly more curvature than is observed in the data, and can be ruled out. In both cases shown for $k$, the comparison between the observations and model predictions indicate a lower limit of $\tau_{*} \gtrsim 10^{9}$ years for clusters in M51.

There may be weak evidence in some galaxies for a turndown at the high mass end, which would suggest that there is a cutoff to the highest mass with which a cluster can form (see, e.g., Portegies Zwart et al. 2010 and references therein). We do not find obvious evidence for such a downturn for intermediate-age clusters in M51. We presented a detailed analysis of the mass function of these clusters in Chandar et al. (2011), where we compared the results of fitting a simple power law with those of a Schechter function, and found that a power law provided just as good of a fit as a Schechter function, and that any upper mass cutoff must be higher than $M_{C} \gtrsim 2 \times 10^{5} M_{\odot}$.

The shape of the cluster age distribution also appears to be fairly similar in many galaxies, i.e., declines more or less continuously starting at young ages, with no obvious 
dependence on the mass of the clusters (see Fall \& Chandar 2012 and references therein). In this work, we find that the results for M51 are reasonably well described by a power law with index $\gamma=-0.65 \pm 0.15$. This is similar to the age distributions of clusters found in several other star-forming galaxies, including spirals, starbursts, and mergers, where values of $\gamma$ between -0.5 and -1.0 have been found (Fall \& Chandar 2012).

The age distribution reflects the combined formation and disruption histories of the clusters. If the formation history of clusters in M51 has been more or less constant over the last several hundred Myr, as might be expected for large spiral galaxies, then the age distribution reflects mostly the disruption history of the clusters, and clusters appear to disrupt at a rate that is approximately independent of their initial mass, with nearly $\approx 80 \%$ (i.e., $1-10^{-0.65}=0.78$ ) dissolving each decade in age. If there was an increase in the cluster formation rate associated with the dynamical encounter of M51 with it's neighboring galaxy NGC 5195 approximately 100-250 Myr ago as suggested by Hwang \& Lee (2010), then the actual dissolution rate of clusters would be even higher, since a higher rate of cluster formation would artificially raise the observed points in this age range relative to the younger data points.

The results for M51 add to a growing body of results that suggest that the mass and age distributions of star clusters in different galaxies are quite similar. While this is true on galaxy scales, these distributions may not be the same in all parts of all galaxies.

The size distribution of clusters in two different intervals of mass $(\log M=4.5-4.8, \quad$ and $\log M>4.8)$ and age $\left(\log \left(\tau \mathrm{yr}^{-1}\right)<7.0\right.$ and $\left.\log \left(\tau \mathrm{yr}^{-1}\right)=8.0-8.6\right)$ suggests that either more compact clusters are destroyed faster than less compact ones, or that clusters in M51 physically expand, by a factor of $\approx 3-4$ on average for their half-light radii, over the first few hundred million years. We believe that it is more likely that initially compact clusters expand rather than being preferentially disrupted. Several processes have been suggested to cause clusters to expand. We note that there does not appear to be any obvious mass dependence to the expansion rate in our sample.

There are a number of physical mechanisms that can cause clusters to expand, and different processes operate on different timescales. The earliest expansion is likely driven by the expulsion of leftover natal gas from very young clusters. Stars are left with a too large velocity dispersion for their new potential, and the cluster expands as it attempts to find a new equilibrium (Hills 1980; Goodwin \& Bastian 2006). The amount of expansion depends on details of the gas expulsion (e.g., timescale, speed and fraction of gas that is lost, etc.), but $\mathrm{N}$-body simulations suggest that surviving clusters typically expand by a factor of 3-4 over time (Baumgardt \& Kroupa 2007). This typical expansion is consistent with the evolution of sizes that we measure for clusters in M51.

Another process that leads to the expansion of clusters over time is the orbital decay of stellar mass black holes as they accumulate in the cluster center, which can heat the stellar background and create a central core. However, simulations suggest that this occurs on timescales of several hundred Myr and longer, so is unlikely to be the dominant mechanism driving the observed expansion. It is possible, however, that the accumulation of massive stars at cluster centers, within a few Myr after formation, drives some expansion (Merritt et al. 2004). Stellar evolution-driven mass-loss is strong at these young ages, and can also inject sufficient energy to expand clusters (e.g., Fukushige \& Heggie 1995; Gieles et al. 2010).

\section{SUMMARY}

We have used $U B V I, \mathrm{H} \alpha$ images taken with the ACS and WFPC2 cameras on board the HST to identify compact star clusters in the nearby grand-design spiral galaxy M51, and presented a new catalog of 3816 clusters. We estimated the masses and ages of the clusters by comparing the measured photometry with predictions from the population synthesis models of G. Bruzual \& S. Charlot (2007, private communication), and compared the age results for different filter combinations with those determined by Bastian et al. (2008) from integrated spectroscopy for a subset of the clusters. We concluded that the $U B V I, \mathrm{H} \alpha$ combination gives the best results, but that $B V I, \mathrm{H} \alpha$ also returns reasonably good age determinations. The $U B V I$ filter set performed more poorly than either of the two combinations that included the narrowband filter.

The mass function of the clusters at different ages (up to $\tau \approx 400 \mathrm{Myr}$ ) were well described by a single power law, $d N / d M \propto M^{\beta}$, with $\beta \approx-2$, and no obvious bends or breaks at either the high or low mass end. We used the shapes of the CMFs to place lower limits on the typical timescale for the disruption of a $10^{4} M_{\odot}$ cluster, and on any exponential cutoff at the high mass end.

The age distribution of the clusters at different masses could also be described by a power law, $d N / \tau \propto \tau^{\gamma}$, with $\gamma \approx-0.6$. Assuming that the cluster formation history has not been steadily increasing from the past to the present, this suggests that the disruption of the clusters is the primary physical mechanism responsible for the observed shape of the cluster age distribution, and that clusters start disrupting soon after they form, and at a rate that does not depend strongly on their initial mass.

Clusters in M51 show a strong evolution in their size over time, with the typical $R_{\text {eff }}$ of a massive cluster increasing by a factor of $\approx 3-4$ over the first few hundred Myr.

The large cluster sample presented here, with well constrained ages, masses, and sizes, is ideal for establishing trends in cluster properties with galactocentric radius, azimuth, and environment. We will report on some interesting trends in the properties of clusters in different dynamical environments, including the bulge and spiral arms of M51, in an upcoming work.

R.C. is grateful for support from NSF through CAREER award 0847467 and from NASA through grant GO-10501-01A from STScI, which is operated by AURA, Inc., under NASA contract NAS5-26555.

\section{REFERENCES}

Bastian, N., Adamo, A., Gieles, M., et al. 2011, MNRAS, 417, 6

Bastian, N., Adamo, A., Gieles, M., et al. 2012, MNRAS, 419, 2606

Bastian, N., Gieles, M., Goodwin, S. P., et al. 2008, MNRAS, 389, 223

Bastian, N., Gieles, M., Lamers, H. J. G. L. M., Scheepmaker, R. A., \& de Grijs, R. 2005, A\&A, 431, 905

Baumgardt, H., \& Kroupa, P. 2007, MNRAS, 380, 1589

Boutloukos, S. G., \& Lamers, H. J. G. L. M. 2003, MNRAS, 338, 717

Bruzual, G., \& Charlot, S. 2003, MNRAS, 344, 1000

Calzetti, D., Kinney, A. L., \& Storchi-Bergmann, T. 1994, ApJ, 429, 582 
Calzetti, D., Lee, J. C., Sabbi, E., et al. 2015, AJ, 149, 51

Chabrier, G. 2003, PASP, 115, 763

Chandar, R., Fall, S. M., \& Whitmore, B. C. 2010a, ApJ, 711, 1263

Chandar, R., Fall, S. M., \& Whitmore, B. C. 2015, ApJ, 810, 1

Chandar, R., Whitmore, B. C., Calzetti, D., et al. 2011, ApJ, 727, 88

Chandar, R., Whitmore, B. C., Calzetti, D., \& O'Connell, R. 2014, ApJ, 787, 17

Chandar, R., Whitmore, B. C., Kim, J., et al. 2010b, ApJ, 719, 966

Chandar, R., Whitmore, B. C., \& Lee, M. G. 2004, ApJ, 611, 220

de Grijs, R., \& Anders, P. 2006, MNRAS, 366, 295

Dolphin, A. 2000, PASP, 112, 1397

Fall, S. M., \& Chandar, R. 2012, ApJ, 752, 96

Fall, S. M., Chandar, R., \& Whitmore, B. C. 2005, ApJL, 631, L13

Fall, S. M., Chandar, R., \& Whitmore, B. C. 2009, ApJ, 704, 453

Feldmeier, J., Ciardullo, R., \& Jacoby, G. H. 1997, ApJ, 479, 231

Fitzpatrick, E. L. 1999, PASP, 111, 63

Fukushige, T., \& Heggie, D. C. 1995, MNRAS, 276, 206

Gieles, M., Bastian, N., Lamers, H. J. G. L. M., \& Mout, J. N. 2005, A\&A, 441, 949

Gieles, M., Baumgardt, H., Heggie, D. C., \& Lamers, H. J. G. L. M. 2010 , MNRAS, 408, 16

Goodwin, S. P., \& Bastian, N. 2006, MNRAS, 373, 752
Hills, J. G. 1980, ApJ, 235, 986

Holtzmann, J., Burrows, C. J., Casertano, S., et al. 1995, PASP, 107, 1065

Hwang, N., \& Lee, M. G. 2010, ApJ, 709, 411

King, I. 1966, AJ, 71, 64

Lamers, H. J. G. L. M., Gieles, M., \& Portegies Zwart, S. F. 2005, A\&A, 429, L173

Larsen, S. S. 1999, A\&AS, 139, 393

Lee, M. G., Chandar, R., \& Whitmore, B. C. 2005, AJ, 130, 2128

Maiz Apellaniz, J., \& Ubeda, L. 2005, ApJ, 629, 873

Merritt, D., Piatek, S., Portegies Zwart, S., \& Mesendorf, M. 2004, ApJ, 608, 25

Portegies Zwart, S. F., McMillan, S. L. W., \& Gieles, M. 2010, ARA\&A, 48, 431

Ryon, J. E., Bastian, N., Adamo, A., et al. 2015, MNRAS, 452, 525

Salpeter, E. 1955, ApJ, 121, 161

Scheepmaker, R. A., Haas, M. R., Gieles, M., et al. 2007, A\&A, 469, 925

Scheepmaker, R. A., Lamers, H. J. G. L. M., Anders, P., \& Larsen, S. S. 2009 , A\&A, 494, 81

Sirianni, M., Jee, M. J., Bentez, N., et al. 2005, PASP, 117, 1049

Vinko, J., Takats, K., Szalai, T., et al. 2012, A\&A, 540, 93

Whitmore, B. C., Chandar, R., \& Fall, S. M. 2007, AJ, 133, 1067

Whitmore, B. C., Chandar, R., Schweizer, F., et al. 2010, AJ, 140, 75 